\title{
Early-type dwarf galaxies in the M81 group
}

\author{
Sophia Lianou${ }^{1}$, Eva K. Grebel ${ }^{1} \&$ Andreas Koch ${ }^{2}$ \\ ${ }^{1}$ Astronomisches Rechen-Institut, University of Heidelberg, D-69120, Heidelberg, Germany \\ email: lianou@ari.uni-heidelberg.de \\ ${ }^{2}$ University of Leicester, University Road, LE1 7RH Leicester, UK
}

\begin{abstract}
The M81 group is a highly interacting group consisting of a few large galaxies and about 40 dwarfs of both early- and late-type, thus making it an important nearby laboratory to study environmental effects and the role of interactions in the formation and evolution of dwarf galaxies. We are studying the resolved stellar populations of the early-type dwarf galaxies in this group with available HST/ACS data. We will show results on the metallicity distribution functions and on the potential presence of population gradients for these dwarf galaxies.
\end{abstract}

Keywords. galaxies: dwarf, galaxies: evolution, galaxies: stellar content, M81 Group

\section{Introduction}

The M81 group is a nearby galaxy group at a mean distance of $\sim 3.7 \mathrm{Mpc}$ (Karachentsev et al. 2002). It contains three large galaxies and about 40 dwarf galaxies, with the addition of $\sim 12$ new dwarf candidates (Chiboucas et al. 2009). The three-dimensional spatial distribution of the galaxies in the M81 group is shown in Karachentsev et al. 2002 (Fig. 6). While the M81 group is similar to the Local Group (LG) in many ways, in terms of the overall morphological galaxy content and binary structure, it differs substantially, since the M81 group is a strongly interacting group, with its three primary galaxies, namely M 81, M 82 and NGC 3077, being connected by extended HI streams (Appleton et al. 1981; Yun et al. 1994).

We present here results on the properties of nine dwarf spheroidal (dSph) galaxies in the M81 group based on available imaging data from the Hubble Space Telescope (HST)/Advanced Camera for Surveys (ACS). Apart from characterizing the individual galaxies, we ultimately want to explore to what extent environment affects galaxy evolution.

\section{Data and Analysis}

We use available HST/ACS archival data to construct the color-magnitude diagrams (CMDs) for the nine dSphs. We work in the HST/ACS filter system. As an example here, we show in Fig. 1, left, the CMD for DDO 71. Metallicity distribution functions (MDFs) were calculated, and individual metallicities were assigned to each star within the bounding box, shown in Fig. 1 for the case of DDO 71, using linear interpolation between Dartmouth isochrones (Dotter et al. 2008) with a fixed age of 12.5 Gyr. We choose this age under the assumption of the red giant branch (RGB) consisting of mainly old stellar populations within the age range of 10 Gyr to 13 Gyr. The assumption of predominantly old ages is supported by the CMD morphology. Moreover, if the properties of the dSphs in the LG may be used as a guide, most dSphs are dominated by old populations (e.g., Grebel 2001; Grebel \& Gallagher 2004). 
Table 1. Global properties of the studied dSphs.

\begin{tabular}{lcccc}
\hline Galaxy & $N_{*}$ & $\begin{array}{c}M_{V} \\
(\mathrm{mag}) \\
(1)\end{array}$ & $\begin{array}{c}\langle[\mathrm{Fe} / \mathrm{H}]\rangle_{w} \\
(\mathrm{dex})\end{array}$ & $\begin{array}{c}\sigma_{\langle[\mathrm{Fe} / \mathrm{H}]\rangle_{w}} \\
(\mathrm{dex})\end{array}$ \\
\hline KDG61 & 53543 & -13.87 & -1.53 & 0.24 \\
KDG64 & 38012 & -13.43 & -1.57 & 0.23 \\
DDO71 & 37291 & -13.22 & -1.56 & 0.24 \\
F12D1 & 39519 & -12.84 & -1.43 & 0.34 \\
DDO78 & 21073 & -12.83 & -1.36 & 0.20 \\
DDO44 & 19357 & -12.56 & -1.67 & 0.19 \\
IKN & 14600 & -11.51 & -1.12 & 0.07 \\
F6D1 & 14260 & -11.46 & -1.48 & 0.43 \\
HS117 & 4596 & -11.31 & -1.37 & 0.14 \\
\hline
\end{tabular}

We opted for Dartmouth isochrones since these models were shown to provide an excellent fit to the full extent of the CMDs of intermediate-age and old simple stellar populations and to yield very good agreement with spectroscopically determined metallicities (see Glatt et al. 2008a, 2008b). This method is similar to the often-employed technique of interpolating between empirical globular cluster RGB ridge lines or "fiducials" (e.g., Sarajedini et al. 2002).

\section{Results}

The resulting photometric metallicities range from -2.50 to -0.50 dex with large spreads. The error-weighted mean values of the metallicities are listed in the Table 1 , column (4), along with the corresponding uncertainties, column (5), for each galaxy, column (1). In the same Table, we list in column (2) the number of stars detected after applying all the photometric cuts, and in column (3) the absolute visual magnitude. The large spread in $[\mathrm{Fe} / \mathrm{H}]$ is similar to what is observed in LG dSphs (Grebel et al. 2003).
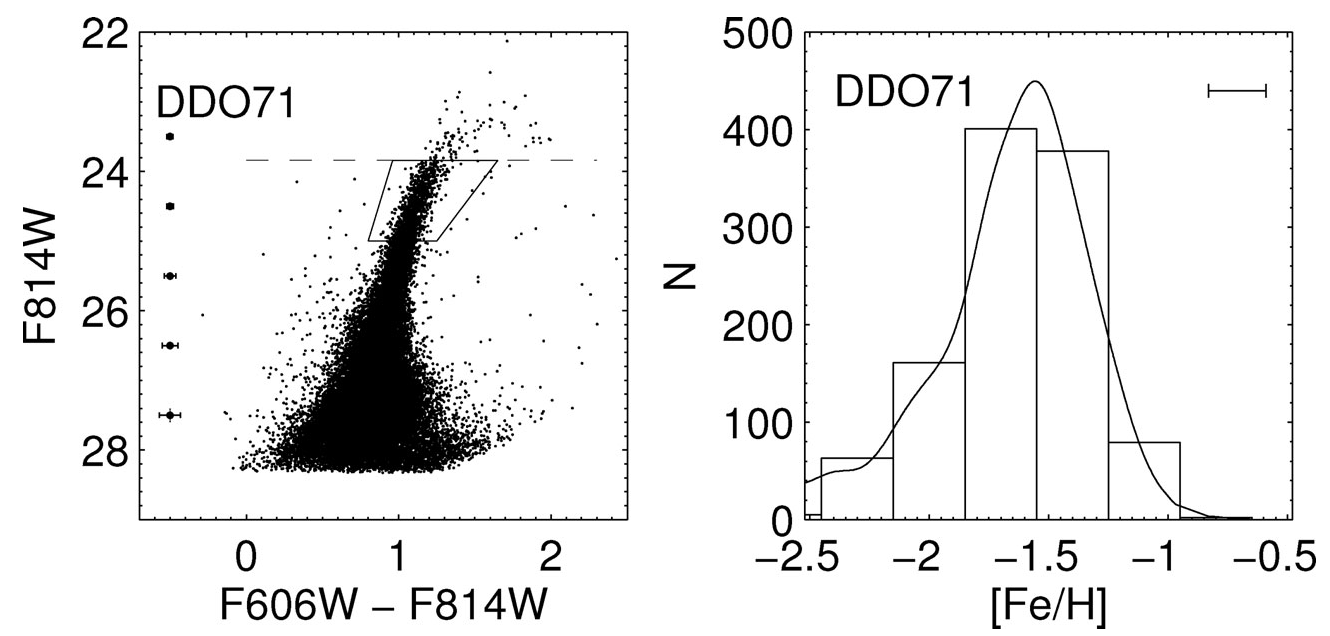

Figure 1. Left panel: Color-magnitude diagram for DDO 71. The dashed line indicates the location of the TRGB. The stars that were selected to construct their metallicity distribution functions are within the solid bounding box. The error bars show the photometric errors. Right panel: Metallicity distribution function for DDO 71. The solid line shows the convolution with the errors in metallicity. The error bar shows the intrinsic $1 \sigma$ dispersion. 
As an example, the MDF for DDO 71 is shown in Fig. 1, right. The majority of the MDFs of the studied dSphs show a more gradual rise from lower to higher metallicities and then a somewhat steeper fall-off. Similar MDF shapes have also been inferred from spectroscopic observations in the LG (e.g., Koch et al. 2006, 2007a, b; Helmi et al. 2006. These MDFs' shapes are due to the action of intense galactic winds (Lanfranchi \& Matteucci 2007) or the effects of extended star formation and inhomogeneous enrichment by SNe Ia (Marcolini et al. 2008).

We show in Fig. 2 the results on the metallicity gradients for DDO 71. The left panel, from top to bottom, shows the radial metallicity distributions, the cumulative distributions, and the radial mean metallicity profiles. The right panel, from top to bottom, shows the spatial distribution of the metal-rich and metal-poor stars, selected as being more metal rich than -1.30 dex and more metal poor than -1.80 dex, respectively, and of the intermediate-age luminous AGB stas, as defined in Caldwell et al. (1998) and Renzini \& Buzzoni (1986). From these distributions we conclude that DDO 71 presents strong metallicity gradients, while in other dwrafs these are not significant. It is interesting to note that the intermediate-age stars appear to be well-mixed with the old stars, a
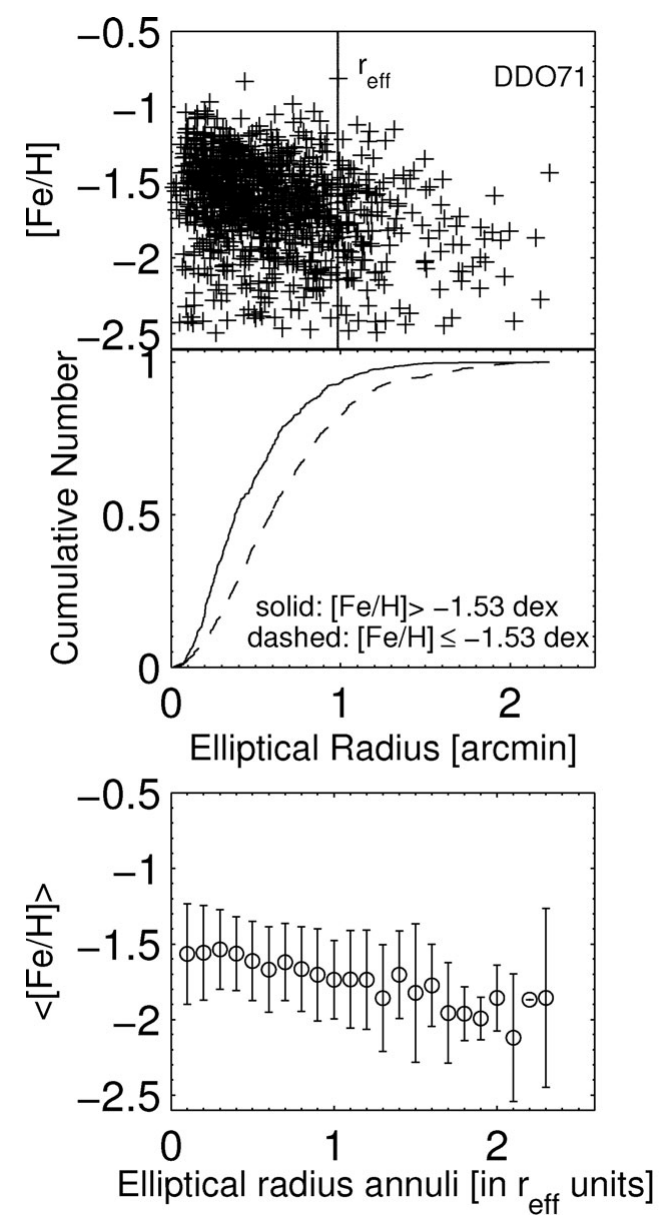

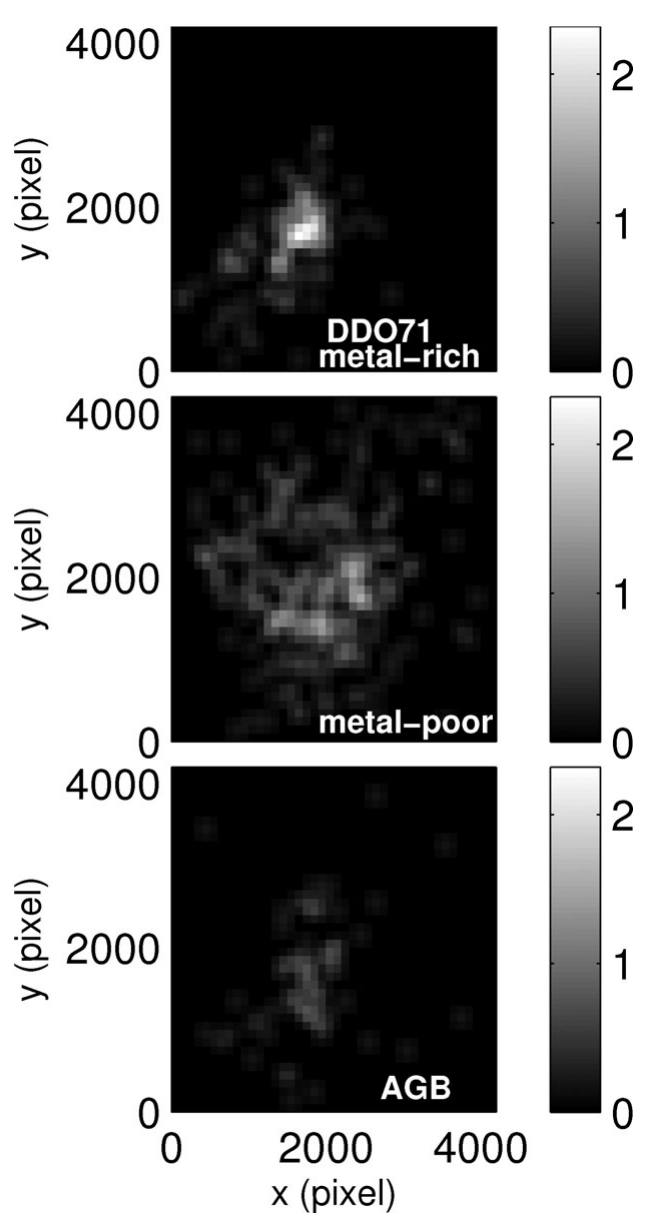

Figure 2. From top to bottom we show, in the left panel, the radial metallicity distribution, cumulative distribution and radial mean metallicity profile for DDO 71, and in the right panel, the spatial distribution of the metal-rich and metal-poor stars, as defined in the text, and of the intermediate-age stars. The unit of the colorbar is stars per $(100 \text { pixel })^{2}$. 
behaviour similar to what is observed for the LG dSphs (Battinelli \& Demers 2004), while the majority of them appear to be more confined to the central regions (Stetson et al. 1998).

\section{Acknowledgements}

SL would like to acknowledge an IAU travel grant to participate to this Symposium. SL and this research were supported within the framework of the Excellence Initiative by the German Research Foundation (DFG) via the Heidelberg Graduate School of Fundamental Physics (HGSFP) (grant number GSC 129/1). AK acknowledges support by an STFC postdoctoral fellowship and by the HGSFP of the the University of Heidelberg.

This research has made use of the NASA/IPAC Extragalactic Database (NED) which is operated by the Jet Propulsion Laboratory, California Institute of Technology, under contract with the National Aeronautics and Space Administration. This research has made use of NASA's Astrophysics Data System Bibliographic Services. This research has made use of SAOImage DS9, developed by Smithsonian Astrophysical Observatory.

\section{References}

Appleton, P. N., Davies, R. D., \& Stephenson, R. J. 1981, MNRAS, 195, 327

Battinelli \& Demers 2004, A\&AA, 417, 479

Caldwell, N., Armandroff, T. E., Da Costa, G. S., \& Seitzer, P. 1998, AJ,115, 535

Chiboucas, K., Karachentsev, I. D., \& Tully, R. B. 2009, AJ, 137, 3009

Dotter, A., Chaboyer, B., Jevremovic, D. et al. 2008, ApJS, 178, 89

Glatt, K. et al. 2008a, AJ, 135, 1106

Glatt, K. et al. 2008b, AJ, 136, 1703

Grebel, E. K. 2001, Ap\&SS Supplement, 277, 231

Grebel, E. K. \& Gallagher, J. S., III 2004, ApJ, 610, L89

Grebel, E. K., Gallagher, J. S. III, \& Harbeck D. 2003, AJ, 125, 1926

Helmi, A. et al. 2006, ApJ,651, L121

Karachentsev, I. D. et al. 2002, A\&SA, 383, 125

Koch, A. et al. 2006, $A J, 131,895$

Koch, A. et al. 2007a, $A J, 133,270$

Koch, A. et al. 2007b, ApJ, 657, 241

Lanfranchi, G. A. \& Matteucci, F. 2007, A\&SA, 468, 927

Marcolini, A., D'Ercole, A., Battaglia, G., \& Gibson, B. K. 2008, MNRAS, 386, 2173

Renzini, A. \& Buzzoni, A. 1986, ASSL, 122, 195

Sarajedini, A. et al. 2002, ApJ, 567, 915

Stetson, P. B., Hesser, J. E., \& Smecker-Hane, T. A. 1998, PASP, 110, 533

Yun, M. S., Ho, P. T. P., \& Lo, K. Y. 1994, Nature, 372, 530 